Review Article

\title{
Interference with the Autophagic Process as a Viral Strategy to Escape from the Immune Control: Lesson from Gamma Herpesviruses
}

\author{
Roberta Santarelli, Marisa Granato, Alberto Faggioni, and Mara Cirone \\ Department of Experimental Medicine, "Sapienza" University of Rome, Viale Regina Elena 324, 00161 Rome, Italy \\ Correspondence should be addressed to Alberto Faggioni; alberto.faggioni@uniromal.it \\ and Mara Cirone; mara.cirone@uniromal.it
}

Received 27 February 2015; Revised 13 April 2015; Accepted 28 April 2015

Academic Editor: Eyad Elkord

Copyright (C) 2015 Roberta Santarelli et al. This is an open access article distributed under the Creative Commons Attribution License, which permits unrestricted use, distribution, and reproduction in any medium, provided the original work is properly cited.

\begin{abstract}
We summarized the most recent findings on the role of autophagy in antiviral immune response. We described how viruses have developed strategies to subvert the autophagic process. A particular attention has been given to Epstein-Barr and Kaposi's sarcoma associated Herpesvirus, viruses studied for many years in our laboratory. These two viruses belong to $\gamma$-Herpesvirus subfamily and are associated with several human cancers. Besides the effects on the immune response, we have described how autophagy subversion by viruses may also concur to the enhancement of their replication and to viral tumorigenesis.
\end{abstract}

\section{Autophagy and the Immune Response}

According to the paradigm of antigen processing by the antigen presenting cells (APC), peptides arising from intracellular proteins are presented via class I MHC molecule. This occurs after they are degraded via proteasome and transported by transporters associated with antigen processing (TAPs). Differently, peptides that originate from extracellular antigens are delivered to the late endosomal/lysosomal compartment, where they are degraded by the lysosomal proteases and then presented in association with class II MHC. In the lysosomes, the invariant chain, which blocks class II MHC, is also degraded, rendering the MHC molecules available for peptide loading. Besides the differences in the sites of origin and processing, peptides presented via class I or II MHC activate different populations of T lymphocytes, which are $\mathrm{CD}^{+}$or $\mathrm{CD} 4^{+}$, respectively [1]. However, exceptions to this model have been described and, for example, dendritic cells (DCs), the most powerful APC [2], are able to present extracellular antigens also via class I MHC. This event, known as cross-presentation, allows DCs to activate both $\mathrm{CD}^{+}$and $\mathrm{CD} 8^{+} \mathrm{T}$ cells, in response to an extracellular antigen [3]. On the other hand, antigens of intracellular origin can be presented also in class II MHC, after being delivered to the lysosomes, through double membrane vesicles, called autophagosomes [4]. Autophagosomes are formed during the induction of autophagy, a self-eating mechanism through which cells recycle their own constituents and survive in stressful conditions. This represents one of the mechanisms through which autophagy may promote the immune response. The autophagic process is regulated by AuTophaGy-related (ATG) genes [5]. Some of them, such as Atgl, Atg11, and Atg13, regulate autophagosome formation; others (e.g., Atg2, Atg9, and Atg18) are required for membrane flow to the expanding phagophore. The vesicle nucleation is instead dependent on the class-III phosphatidylinositol 3 kinase (PtdIns3K) complex formed by Vps34, Vps15, Vps30/Atg6, and Atg14. Atg6, also named Beclin 1, represents a protein with a pivotal role in the autophagy induction [6] and the microtubule-associated protein light chain 3 (LC3), or Atg8, is a marker of the autophagic vacuoles [7]. LC3 is expressed as full-length cytosolic protein and upon autophagy induction is cleaved by Atg 4 to form LC3I, which is then conjugated to phosphatidylethanolamine (PE), generating LC3II. This molecule, whose formation indicates autophagy induction, is associated with the internal and 
external membrane of autophagosome and, by interacting with adaptor molecules, such as p62/SQSTM1, promotes uptake and degradation of both cargo and adaptors into the lysosomes [8]. Indeed, autophagosomes fuse with lysosomes transporting intracellular proteins in the last autophagic steps. Among them, also viral antigens can end up in the lysosomes, in virally infected cells. Here peptides derived from their degradation may be complexed and presented in association with class II MHC molecules. Another important role of autophagosomes is to transport entire viral particles to the endosomal/lysosomal compartment, to be degraded and eventually eliminated, a process known as xenophagy [9]. After entering the cells, pathogens can be directly delivered to the lysosomes through phagosomes. Alternatively, phagosomes as well as free viral particles can be engulfed by the double membrane vesicles of autophagosomes, before reaching the lysosomes. The latter process is under the control of the ATG genes, even if it represents a particular form of autophagy aimed at the antimicrobial defense [10]. In addition, autophagy facilitates the pathogen engagement of intracellular TLRs and the consequent release of cytokines, such as type I IFN [11]. The binding of TLRs as well as other molecules involved in the immune response, for example, CD46, by ligands or pathogens that use them as receptors, also triggers autophagy. This has been reported for EpsteinBarr virus (EBV) that binds TLRs [12] and for measles virus that uses CD46 as receptor [13]. Autophagy has been also shown to contribute to measles virus infectivity [14]. Intriguingly, since CD46 represents the cellular receptor also for human Herpes-virus 6 (HHV6) [15], it would be interesting to investigate the impact on autophagy of CD46 engagement by HHV6. As if the role of autophagy in the immune response was not enough important, it has been recently reported that autophagy is induced by GM-CSF in monocytes and that it stimulates monocyte differentiation into functional macrophages and DCs. Besides that, autophagy also plays an essential role in preventing apoptosis of these cells [16].

\section{Autophagy Manipulation by Viruses}

Among the strategies that allow viruses to escape from the immune control, the impairment of monocyte differentiation into functional DCs represents a common one [17-19]. Based on the recent finding, suggesting that autophagy is involved in monocyte differentiation in macrophages and DCs, the interference with the autophagic process could represent one of the underlying mechanisms responsible for such impairment. This strategy has been recently reported to be exploited by Human Hepatitis C virus (HCV), whose infection of human monocytes results in a reduction of lysosomal cathepsins, in an autophagic block at the late steps, and, as a consequence, in an impairment of DC differentiation [20]. HCV-mediated reduction of lysosomal acidification, as result of cathepsin release, has been reported also in other cell types [21]. However, autophagy can contribute to HCV replication since the autophagosomal membranes can be used for viral production [22]. Given the importance of autophagy in the immune response, it is not surprising that viruses have evolved strategies to interfere with it, in order to avoid their elimination into the lysosomes, impair the production of antiviral cytokines, reduce the presentation of their antigens, and, as described for $\mathrm{HCV}$, alter DC differentiation. This is a must for viruses to persist in the infected host, sometimes with pathological consequences. All the steps of the autophagic process, from the autophagosome formation to the lysosomal degradation of their content [23], can be manipulated by viruses [24, 25] (Figure 1). It has been described that it depends on the virus types, on the phase of their life cycle, and on the host cell that they infect. For example, Human Immunodeficiency Virus [23], a single-stranded RNA lentivirus belonging to the Retroviridae family, is able to induce the initial phases of autophagy by Env, an envelope fusogenic protein. On the other hand, it blocks the late autophagic phases to enhance viral production by expressing Nef, an accessory protein that interferes with the autophagosomal maturation [24, 25]. During the replicative phase of their life cycle, also herpesviruses such as EBV and Kaposi's Sarcoma Human Herpesvirus (KSHV) can promote autophagy and exploit the autophagic machinery to enhance their replication [26-28]. To do so, they promote the first autophagic steps and block the last ones. This strategy allows them to avoid being delivered into the destructive environment of the lysosomes and to usurp the autophagic machinery for viral transportation through the cell cytoplasm $[22,26,29]$. Pathways involved in the autophagy induction, such as PKR/EIF2 alpha and m-Tor, can be also targeted by viruses belonging to the Herpesvirus family, for example, Herpes Simplex virus-1 (HSV-1) [3032] and cytomegalovirus (HCMV) [33]. Also Beclin 1, a key molecule involved in several steps of the autophagic process [6], can be bound and altered in its function by viral proteins such as vBcl2 of KSHV or ICP 34.5 of HSV-1 [3436]. All these strategies allow viruses to block autophagy induction during infection of the host cells. Differently, another Herpesvirus, Varicella-Zoster virus (VZV), has been recently reported to successfully infect target cells without blocking the autophagic process. Indeed ICP 34.5 or US11, the two proteins responsible for the block of autophagy by HSV1, are not present in VZV although both viruses belong to the same subfamily [37].

\section{Herpesviruses}

Herpesviruses are large, double strand DNA viruses having a common particle structure [38]. To date eight human Herpesviruses have been identified and classified into three subfamilies (alpha, beta, and gamma) based on their growth characteristics and tissue tropism [39]. The $\alpha$-subfamily includes the neurotropic viruses Herpes Simplex viruses (HSV) 1 and 2 and Varicella-Zoster virus (VZV). Human cytomegalovirus (HCMV) and the human Herpesviruses 6 and 7 are members of the $\beta$-subfamily, while EBV and KSHV are members of the $\gamma$-subfamily [40]. A common feature shared by all human Herpesviruses is the viral persistence into host and the possibility to undergo two alternative life cycle programs, namely, latency and lytic replication. During latency the viral genome is retained as a circular episome in the nucleus and no viral progeny is produced. Furthermore, in the course of latent infection a limited set of genes is 


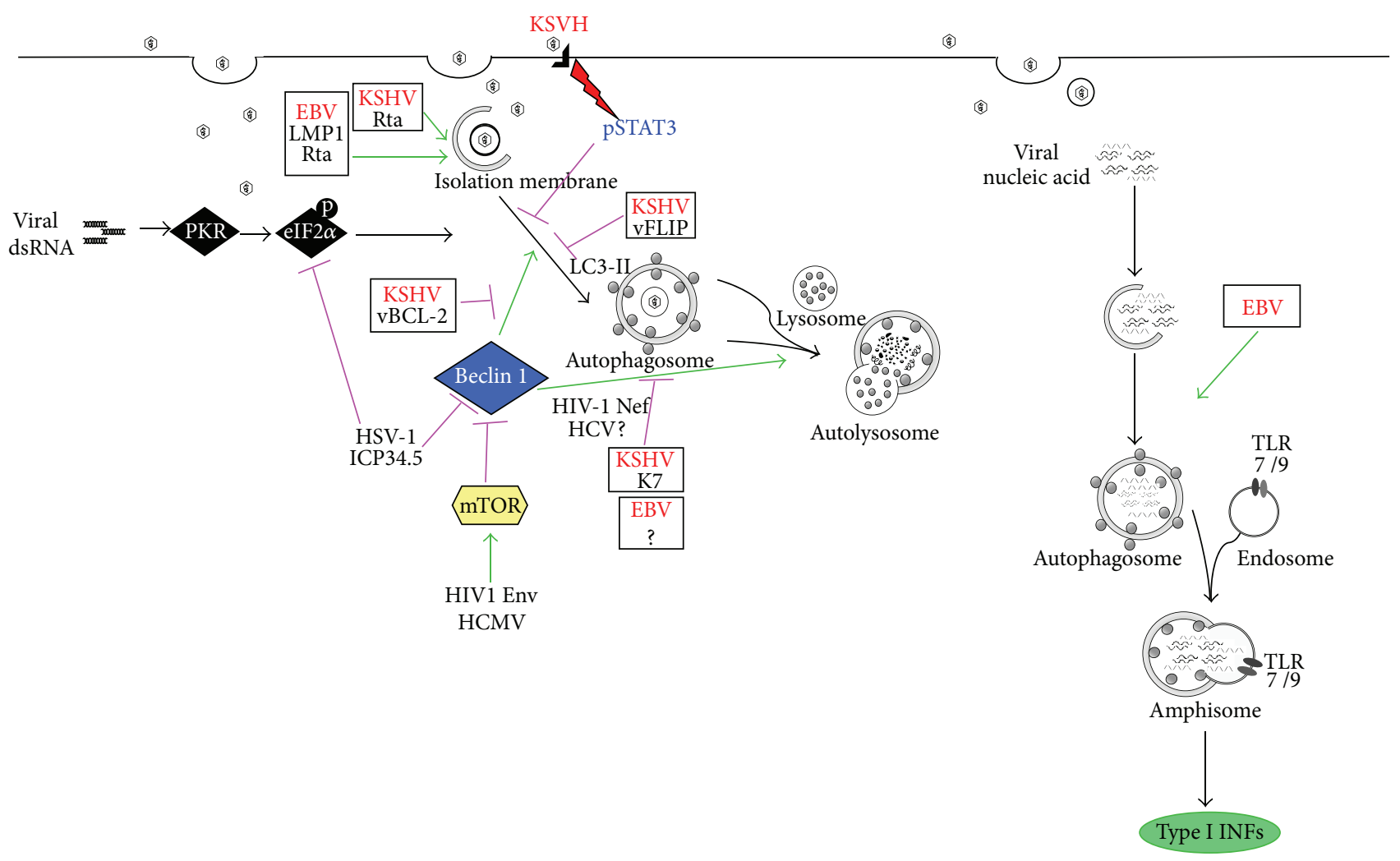

$\longrightarrow$ Induction of the autophagic steps
Inhibition of the autophagic steps

FIGURE 1: Examples of autophagy manipulation by human viruses.

expressed in order to reduce immune recognition. In contrast to latency, the Herpesvirus lytic program is characterized by a regulated cascade of viral gene expression accompanied by viral production and killing of infected cells [23]. Although most Herpesviruses have been reported to interfere with the autophagic process, the next part of this review will focus on the interplay between autophagy and $\gamma$-Herpesviruses, given that our laboratory has for long time worked on the virus-host interaction of these viruses associated with several human cancers.

\section{Epstein-Barr Virus (EBV)}

As for other Herpesviruses, EBV infection can be latent or lytic [41, 42]. During latency the EBV genome is retained as a circular episome in the cell nuclei and upon appropriate stimuli the virus switches into the lytic replication program. It is known that EBV infects primarily human B lymphocytes and epithelial cells; however, its infection can also occur in other cells with a central role in the immune response [43], such as monocytes and DCs $[44,45]$. The in vitro infection of monocytes induces apoptosis and results in an impairment of DC development $[45,46]$, although the underlying mechanisms have not been investigated in these studies. Therefore, the autophagic pathway could be investigated in these cells since, as previously described, a block of autophagy can switch cell differentiation into cell death in monocytes DCs [16]. These are the most powerful cells in the priming of the $\mathrm{CD}^{+} \mathrm{T}$ cell response, playing a pivotal role in control of the EBV infection [47]. Besides cytotoxic T cells, DCs are able to activate a $\mathrm{CD} 4^{+} \mathrm{T}$ cell-mediated immune response against the EBV antigens, such as EBNA1, through the mechanism of cross-presentation [48]. Autophagy has been shown to be essential for class II MHC presentation of EBNA1 protein. The block of autophagy resulted in an accumulation of this protein in the intracellular autophagosomes and, more importantly, in a reduction of EBNA1 recognition by EBNA1 specific $\mathrm{CD}^{+}{ }^{+} \mathrm{T}$ cells [49]. The involvement of autophagy in EBNA 1 presentation was later confirmed by a more recent study [50]. Regarding the EBV interaction with plasmacytoid subpopulation of DCs (pDCs), the main type I IFN producing cells [51], it has been reported that autophagy is essential for IFN release in response to the EBV infection. Autophagy is stimulated by the virus and facilitates its interaction with TLR7 and TLR9 PPRs, both located in the endosomal/lysosomal compartment and essential for EBV recognition by these cells. However, although EBV stimulates autophagy and IFN release, its infection results in an impairment of pDC maturation [12]. Since it has been reported that EBV downregulates TLR 9 in B cells [52] and that this effect is mediated by two EBV proteins, namely, latent membrane protein 1 (LMP1) [53] and BGLF5 [54], it 
would be interesting to investigate TLR9 expression levels in the pDCs EBV-infected versus uninfected control cells.

EBV is associated with several different human cancers of $B$ and epithelial cell origin, such as posttransplant lymphoproliferative disorder (PTLD), Hodgkin and non-Hodgkin lymphomas, nasopharyngeal carcinoma (NPC), and some forms of gastric carcinoma [55]. Besides its strong association with some human cancers, EBV infects and establishes a life-long asymptomatic infection in $95 \%$ of adult healthy population [56], reducing to the minimum or not expressing any protein, in order to escape from the immune recognition. Among the viral antigens, only the EBV latent nuclear antigen 1 (EBNA1) expression is required for the maintenance of the viral episome in the EBV infected human B cells [57] and this is the only protein always expressed in EBV-associated malignancies. This protein has been demonstrated to activate both $\mathrm{CD}^{+} \mathrm{T}$ cells [44], being presented via class II MHC [49] and $\mathrm{CD}^{+} \mathrm{T}$ cells [58], although for long time it has been considered invisible to the immune system [48]. As previously described, autophagy is essential for EBNA1 antigen presentation via class II MHC while, interestingly, it does not seem to play a role in the class II MHC presentation of two other EBV latent nuclear proteins, EBNA2 and EBNA 3C, both expressed only in pathological conditions [59]. The most oncogenic EBV latent protein, LMP1, has been reported to regulate its own expression by inducing or inhibiting autophagy. When LMP1 is highly expressed in B cells, autophagy is stimulated and promotes degradation [60]. Given that LMP1 plays an important role in EBV-induced oncogenesis, the stimulation of autophagy could be used as a strategy to reduce the expression of this protein and consequently to affect the EBV-driven tumorigenesis.

\section{Kaposi's Sarcoma-Associated Herpesvirus (KSHV)}

KSHV or Human Herpesvirus-8 (HHV-8) is the last human $\gamma$-Herpesvirus identified to date [61]. It shows two alternative life cycle programs, latent and lytic [62-64]. Generally, latency is the KSHV default program within $48-72 \mathrm{~h}$ postinfection depending on target cells. In vivo, KSHV mainly infects endothelial and B cells and establishes a lifelong latency in B lymphocytes of infected individuals, escaping from the host immune response [65, 66]. Several KSHV latent and lytic proteins are involved in immune evasion, in preventing apoptosis and blocking autophagy as well as in transformation [67]. KSHV is the etiologic agent of Kaposi's sarcoma (KS) [61], a multifocal angioproliferative disorder arising from KSHV-infected endothelial cell [68], and of lymphoproliferative disorders, such as primary effusion lymphomas (PELs), a non-Hodgkin B cell lymphoma localized in body cavities [66], and the plasma cell variant of multicentric Castleman disease (MCD) [69]. It is important to underline that, although KSHV is detectable in all KS lesions, regardless of disease stage or clinical variant, the virus is necessary but not sufficient for the development of this tumor [67, 68]. Typical of KS are the spindle cells, derived from the endothelial cells. The majority of spindle cells are latently infected by KSHV and only a small percentage of these cells express viral lytic antigens $[68,70]$. However, it is currently believed that lytic replication is necessary to support KS lesion formation and maintenance [68, 71-74]. In addition, viral replication allows the secretion of proinflammatory and/or proangiogenic factors that create the inflammatory microenvironment essential in KS pathogenesis $[68,75]$. Monocytes are among the inflammatory cells found in KS lesions and a study, based on immunohistochemical staining and in situ hybridization, reported that these cells are KSHV infected [76]. The virus has also been detected in peripheral T-cells and macrophages [77, 78].

As other Herpesviruses, KSHV has to cope with innate and adaptive immunity in order to establish a persistent infection in immunocompetent host. Latency is one of the strategies used by KSHV since viral gene expression is reduced. Conversely, during its lytic cycle, a high number of immunogenic proteins are produced [71,79]. The relevance of the equilibrium between KSHV lytic cycle and host immunity is indicated by the increased viral replication and the development of KSHV-related malignancies in immunosuppressed patients [80-82]. About $25 \%$ of KSHV proteins are involved in KSHV immune evasion mechanisms [79] and, although most of these belong to lytic cycle, both latent and lytic proteins are able to hijack innate and adaptive immune response [67]. They can indeed inhibit complementmediated lysis of infected cells and the IFN I signaling, deregulate the inflammatory cytokine/chemokine networks, and interfere with antigen presentation $[67,79,83-85]$. pDCs, monocyte-derived dendritic cells, and monocytes are among the cell types infected by KSHV [19, 86, 87]. As consequence of the infection, a reduction of costimulatory molecules as well as a deregulation of the cytokine release and an impairment of allostimulatory capacity can occur $[19,76,87-$ 90]. In vivo, DC functional impairment has been reported in patients with classical KS [91] and a reduction of pDCs was observed in AIDS-KS in comparison to KS negative HIV-1 infected individuals [92]. Moreover, the number of Langerhans cells is also decreased in KS lesions compared to normal skin [93]. Furthermore, it has been reported that the signal transducer and activator of transcription 3 (STAT3) activation in noninfected monocytes/macrophages leads to a block of autophagy and consequent dysfunction, due to the cytokines released during HIV infection [94]. Interestingly, STAT3 inhibitors are potent autophagy inducers [95]. In this context we showed that KHSV is able to activate STAT3 pathway in DCs by binding to its receptor on these cells, namely, the type II C-type lectin, dendritic cell-specific ICAM-3 grabbing nonintegrin (DC-SIGN; CD209) $[87,96]$. Besides, we showed that STAT3 activation led to a block of the autophagic flux, as demonstrated by the reduced expression of LC3II and the increased level of p62 [96]. In addition, looking for a possible mechanism responsible for the block of autophagy mediated by STAT3 activation in DCs exposed to KSHV, we found an upregulation of Mcl-1 $[96,97]$. This is one of the proteins able to bind and sequester Beclin 1, hampering its essential role in autophagosome formation. Remarkably, STAT3 inhibition by AG490 was able to prevent the effects on p62, LC3II, and Mcl-1 [96]. In agreement, a previous paper has shown that the inhibition of STAT3 by 
Sorafenib resulted in a downregulation of Mcl-1, disruption of Beclin 1-Mcl-1 complex, and reversion of the autophagic block in hepatocarcinoma cells [98]. Of note, the autophagic block occurred also in the presence of UV-inactivated KSHV [96], indicating that neither vBCL2 nor vFLIP expression was required $[34,96,99,100]$. Concomitantly to the autophagic block induced by KSHV-mediated STAT3 activation, we also observed a reduction of IL12p70 release in response to LPS stimulation and a higher production of IL-10, IL6 , and IL-23 [96]. This cytokine pattern skews the Th1/Th2 profile towards Th2 [88] and/or Th17 [101], promoting immunosuppression and inflammation. Therefore, STAT3 activation could be one of the molecular mechanisms underlying KSHV-mediated immunosuppression in DCs. Indeed, STAT3 activation correlates with an immunosuppressive phenotype and DC dysfunction in the KS microenvironment [102] and in the peripheral blood of tumor bearing patients $[103,104]$. Several papers have investigated the interplay between KSHV and the autophagic pathway [79]. During the latent phase of its life-cycle, KSHV expresses the FADDlike interleukin-1 beta-converting enzyme inhibitory protein (v-FLIP), a truncated homolog of the cellular FLIP, which besides having an antiapoptotic activity $[105,106]$ has been recently shown to block the autophagic flux. It competes with LC3 for binding to ATG3, thereby preventing LC3 binding and processing during autophagosome biogenesis [100]. Furthermore, the inhibition of vFLIP binding to ATG3 reduced the size of KSHV positive tumor in mice [100]. It has also been demonstrated that v-FLIP suppression of autophagy counteracts v-cyclin-induced cellular senescence $[107,108]$. v-cyclin is a KSHV latent protein that deregulates cell cycle, causes aberrant host DNA replication, and triggers the DNA damage responses (DDRs) [109]. Besides vFLIP, two KSHV proteins expressed during the lytic cycle, $\mathrm{vBcl} 2$ and $\mathrm{K} 7$, can interfere with autophagy. $\mathrm{vBcl} 2$ is a homolog of cellular Bcl-2 that inhibits both apoptosis $[99,110]$ and autophagy $[34,35]$. As its cellular counterpart, $\mathrm{vBcl} 2$ negatively regulates autophagy by binding Beclin 1 [34]. Always within the context of the lytic cycle, recent evidence suggests that KSHV K7 protein prevents autophagosome maturation, by interacting with Rubicon autophagic protein [111]. K7 is involved in apoptosis suppression [112,113] while Rubicon is a subunit of the Beclin 1/UVRAG/Vps34 autophagy complex, which regulates the autophagosome maturation and the endocytic trafficking [114-116]. As shown by authors, K7 transfection in epithelial cell lines promoted a greater interaction between Rubicon and Beclin 1/UVRAG/Vps34 complex resulting in a block of Vps34 enzymatic activity and, consequently, of autophagosome maturation [111]. Interestingly, during the lytic cycle KSHV exploits autophagy to enhance its reactivation mediated by Rta immediate lytic protein [28]. Altogether, the data here reviewed suggest that KSHV has evolved several strategies to circumvent autophagy-mediated immune responses, to persist in infected hosts. Moreover, the autophagic block during latency and the autophagy induction during the lytic cycle may contribute to the pathogenesis of KSHV associated malignancies.

\section{Concluding Remarks}

A better understanding of how autophagy manipulation could influence antiviral immune response and control virusinduced tumorigenesis might help to discover strategies improving the outcome of the treatments of virus-associated malignancies. Moreover, since autophagy is activated and is involved in EBV and KSHV replication [20, 27], its manipulation could also affect the viral particle release that plays a role in $\gamma$-Herpesvirus-associated cancers.

\section{Disclosure}

Alberto Faggioni and Mara Cirone are co-last authors.

\section{Conflict of Interests}

The authors declare that there is no conflict of interests regarding the publication of this paper.

\section{References}

[1] C. Watts and S. Powis, "Pathways of antigen processing and presentation," Reviews in Immunogenetics, vol. 1, no. 1, pp. 6074, 1999.

[2] P. Guermonprez, J. Valladeau, L. Zitvogel, C. Théry, and S. Amigorena, "Antigen presentation and $\mathrm{T}$ cell stimulation by dendritic cells," Annual Review of Immunology, vol. 20, pp. 621667, 2002.

[3] A. R. Mantegazza, J. G. Magalhaes, S. Amigorena, and M. S. Marks, "Presentation of phagocytosed antigens by MHC Class I and II," Traffic, vol. 14, no. 2, pp. 135-152, 2013.

[4] C. Münz, "Antigen processing for MHC class II presentation via autophagy," Frontiers in Immunology, vol. 3, article 9, 2012.

[5] Y. Feng, D. He, Z. Yao, and D. J. Klionsky, "The machinery of macroautophagy," Cell Research, vol. 24, no. 1, pp. 24-41, 2014.

[6] X. H. Liang, S. Jackson, M. Seaman et al., "Induction of autophagy and inhibition of tumorigenesis by beclin 1," Nature, vol. 402, no. 6762, pp. 672-676, 1999.

[7] Y. Kabeya, N. Mizushima, T. Ueno et al., "LC3, a mammalian homologue of yeast Apg8p, is localized in autophagosome membranes after processing," The EMBO Journal, vol. 19, no. 21, pp. 5720-5728, 2000.

[8] S. Pankiv, T. H. Clausen, T. Lamark et al., "p62/SQSTM1 binds directly to Atg8/LC3 to facilitate degradation of ubiquitinated protein aggregates by autophagy," Journal of Biological Chemistry, vol. 282, no. 33, pp. 24131-24145, 2007.

[9] T. Wileman, "Autophagy as a defence against intracellular pathogens," Essays in Biochemistry, vol. 55, no. 1, pp. 153-163, 2013.

[10] K. A. Bauckman, N. Owusu-Boaitey, and I. U. Mysorekar, "Selective autophagy: xenophagy," Methods, vol. 75, pp. 120-127, 2015.

[11] M. A. Delgado, R. A. Elmaoued, A. S. Davis, G. Kyei, and V. Deretic, "Toll-like receptors control autophagy," The EMBO Journal, vol. 27, no. 7, pp. 1110-1121, 2008.

[12] M. Severa, E. Giacomini, V. Gafa et al., "EBV stimulates TLRand autophagy-dependent pathways and impairs maturation in plasmacytoid dendritic cells: implications for viral immune escape," European Journal of Immunology, vol. 43, no. 1, pp. 147158, 2013. 
[13] P.-E. Joubert, G. Meiffren, I. P. Grégoire et al., "Autophagy induction by the pathogen receptor CD46," Cell Host and Microbe, vol. 6, no. 4, pp. 354-366, 2009.

[14] C. Richetta, I. P. Grégoire, P. Verlhac et al., "Sustained autophagy contributes to measles virus infectivity," PLoS Pathogens, vol. 9, no. 9, Article ID e1003599, 2013.

[15] F. Santoro, P. E. Kennedy, G. Locatelli, M. S. Malnati, E. A. Berger, and P. Lusso, "CD46 is a cellular receptor for human herpesvirus 6," Cell, vol. 99, no. 7, pp. 817-827, 1999.

[16] Y. Zhang, M. J. Morgan, K. Chen, S. Choksi, and Z.-G. Liu, "Induction of autophagy is essential for monocyte-macrophage differentiation," Blood, vol. 119, no. 12, pp. 2895-2905, 2012.

[17] A. Alcami and U. H. Koszinowski, "Viral mechanisms of immune evasion," Immunology Today, vol. 21, no. 9, pp. 447$455,2000$.

[18] A. Iannello, O. Debbeche, E. Martin, L. H. Attalah, S. Samarani, and A. Ahmad, "Viral strategies for evading antiviral cellular immune responses of the host," Journal of Leukocyte Biology, vol. 79, no. 1, pp. 16-35, 2006.

[19] M. Cirone, G. Lucania, P. Bergamo, P. Trivedi, L. Frati, and A. Faggioni, "Human herpesvirus 8 (HHV-8) inhibits monocyte differentiation into dendritic cells and impairs their immunostimulatory activity," Immunology Letters, vol. 113, no. 1, pp. 4046, 2007.

[20] M. Granato, V. Lacconi, M. Peddis et al., "Hepatitis C virus present in the sera of infected patients interferes with the autophagic process of monocytes impairing their in-vitro differentiation into dendritic cells," Biochimica et Biophysica ActaMolecular Cell Research, vol. 1843, no. 7, pp. 1348-1355, 2014.

[21] S. Taguwa, H. Kambara, N. Fujita et al., "Dysfunction of autophagy participates in vacuole formation and cell death in cells replicating hepatitis C virus," Journal of Virology, vol. 85, no. 24, pp. 13185-13194, 2011.

[22] D. Sir, C. F. Kuo, Y. Tian et al., "Replication of hepatitis C virus RNA on autophagosomal membranes," The Journal of Biological Chemistry, vol. 287, no. 22, pp. 18036-18043, 2012.

[23] D. J. Klionsky, F. C. Abdalla, H. Abeliovich et al., "Guidelines for the use and interpretation of assays for monitoring autophagy," Autophagy, vol. 8, no. 4, pp. 445-544, 2012.

[24] S. B. Kudchodkar, Y. Yu, T. G. Maguire, and J. C. Alwine, "Human cytomegalovirus infection induces rapamycin-insensitive phosphorylation of downstream effectors of mTOR kinase," Journal of Virology, vol. 78, no. 20, pp. 11030-11039, 2004.

[25] H. J. Kim, S. Lee, and J. U. Jung, "When autophagy meets viruses: a double-edged sword with functions in defense and offense," Seminars in Immunopathology, vol. 32, no. 4, pp. 323341, 2010.

[26] M. Granato, R. Santarelli, A. Farina et al., "Epstein-barr virus blocks the autophagic flux and appropriates the autophagic machinery to enhance viral replication," Journal of Virology, vol. 88, no. 21, pp. 12715-12726, 2014.

[27] C. H. Hung, L. W. Chen, W. H. Wang et al., "Regulation of autophagic activation by Rta of Epstein-Barr virus via the extracellular signal-regulated kinase pathway," Journal of Virology, vol. 88, no. 20, pp. 12133-12145, 2014.

[28] H.-J. Wen, Z. Yang, Y. Zhou, and C. Wood, "Enhancement of autophagy during lytic replication by the Kaposi's sarcomaassociated herpesvirus replication and transcription activator," Journal of Virology, vol. 84, no. 15, pp. 7448-7458, 2010.

[29] H. Nowag and C. Munz, "Diverting autophagic membranes for exocytosis," Autophagy, vol. 11, no. 2, pp. 425-427, 2015.
[30] B. He, M. Gross, and B. Roizman, "The $\gamma_{1} 34.5$ protein of herpes simplex virus 1 complexes with protein phosphatase $1 \alpha$ to dephosphorylate the $\alpha$ subunit of the eukaryotic translation initiation factor 2 and preclude the shutoff of protein synthesis by double-stranded RNA-activated protein kinase," Proceedings of the National Academy of Sciences of the United States of America, vol. 94, no. 3, pp. 843-848, 1997.

[31] S. J. Child, M. Hakki, K. L. De Niro, and A. P. Geballe, "Evasion of cellular antiviral responses by human cytomegalovirus TRS1 and IRS1," Journal of Virology, vol. 78, no. 1, pp. 197-205, 2004.

[32] M. Hakki, E. E. Marshall, K. L. De Niro, and A. P. Geballe, "Binding and nuclear relocalization of protein kinase $\mathrm{R}$ by human cytomegalovirus TRS1," Journal of Virology, vol. 80, no. 23, pp. 11817-11826, 2006.

[33] M. Chaumorcel, M. Lussignol, L. Mouna et al., "The human cytomegalovirus protein TRS1 inhibits autophagy via its interaction with Beclin 1," Journal of Virology, vol. 86, no. 5, pp. 25712584, 2012.

[34] S. Pattingre, A. Tassa, X. Qu et al., "Bcl-2 antiapoptotic proteins inhibit Beclin 1-dependent autophagy," Cell, vol. 122, no. 6, pp. 927-939, 2005.

[35] C. Liang, E. Xiaofei, and J. U. Jung, "Downregulation of autophagy by herpesvirus Bcl-2 homologs," Autophagy, vol. 4, no. 3, pp. 268-272, 2008.

[36] A. Orvedahl, D. Alexander, Z. Tallóczy et al., "HSV-1 ICP34.5 confers neurovirulence by targeting the Beclin 1 autophagy protein," Cell Host and Microbe, vol. 1, no. 1, pp. 23-35, 2007.

[37] E. M. Buckingham, J. E. Carpenter, W. Jackson, L. Zerboni, A. M. Arvin, and C. Grose, "Autophagic flux without a block differentiates varicella-zoster virus infection from herpes simplex virus infection," Proceedings of the National Academy of Sciences, vol. 112, no. 1, pp. 256-261, 2015.

[38] F. Liu and Z. H. Zhou, "Comparative virion structures of human herpesviruses," in Human Herpesviruses: Biology, Therapy, and Immunoprophylaxis, A. Arvin, G. Campadelli-Fiume, E. Mocarski et al., Eds., Cambridge University Press, Cambridge, UK, 2007.

[39] A. J. Davison, "Overview of classification," in Human Herpesviruses: Biology, Therapy, and Immunoprophylaxis, A. Arvin, G. Campadelli-Fiume, E. Mocarski et al., Eds., Cambridge University Press, Cambridge, UK, 2007.

[40] R. Longnecker and F. Neipel, "Introduction to the human gamma-herpesviruses," in Human Herpesviruses: Biology, Therapy, and Immunoprophylaxis, A. Arvin, G. Campadelli-Fiume, E. Mocarski et al., Eds., Cambridge University Press, Cambridge, UK, 2007.

[41] T. Tsurumi, M. Fujita, and A. Kudoh, "Latent and lytic EpsteinBarr virus replication strategies," Reviews in Medical Virology, vol. 15, no. 1, pp. 3-15, 2005.

[42] W. Amon and P. J. Farrell, "Reactivation of Epstein-Barr virus from latency," Reviews in Medical Virology, vol. 15, no. 3, pp. 149156, 2005.

[43] O. Chijioke, T. Azzi, D. Nadal, and C. Münz, "Innate immune responses against Epstein Barr virus infection," Journal of Leukocyte Biology, vol. 94, no. 6, pp. 1185-1190, 2013.

[44] M. Savard, C. Bélanger, M. Tardif, P. Gourde, L. Flamand, and J. Gosselin, "Infection of primary human monocytes by EpsteinBarr virus," Journal of Virology, vol. 74, no. 6, pp. 2612-2619, 2000.

[45] L. Li, D. Liu, L. Hutt-Fletcher, A. Morgan, M. G. Masucci, and V. Levitsky, "Epstein-Barr virus inhibits the development 
of dendritic cells by promoting apoptosis of their monocyte precursors in the presence of granulocyte macrophage-colonystimulating factor and interleukin-4," Blood, vol. 99, no. 10, pp. 3725-3734, 2002.

[46] A. O. Guerreiro-Cacais, L. Q. Li, D. Donati et al., "Capacity of Epstein-Barr virus to infect monocytes and inhibit their development into dendritic cells is affected by the cell type supporting virus replication," Journal of General Virology, vol. 85, no. 10, pp. 2767-2778, 2004.

[47] K. Bickham, K. Goodman, C. Paludan et al., "Dendritic cells initiate immune control of epstein-barr virus transformation of B lymphocytes in vitro," Journal of Experimental Medicine, vol. 198, no. 11, pp. 1653-1663, 2003.

[48] C. Münz, K. L. Bickham, M. Subklewe et al., "Human CD4 ${ }^{+}$ T lymphocytes consistently respond to the latent EpsteinBarr virus nuclear antigen EBNA1," Journal of Experimental Medicine, vol. 191, no. 10, pp. 1649-1660, 2000.

[49] C. Paludan, D. Schmid, M. Landthaler et al., "Endogenous MHC class II processing of a viral nuclear antigen after autophagy," Science, vol. 307, no. 5709, pp. 593-596, 2005.

[50] C. S. Leung, T. A. Haigh, L. K. Mackay, A. B. Rickinson, and G. S. Taylor, "Nuclear location of an endogenously expressed antigen, EBNA1, restricts access to macroautophagy and the range of CD4 epitope display," Proceedings of the National Academy of Sciences of the United States of America, vol. 107, no. 5, pp. 21652170, 2010.

[51] B. Reizis, A. Bunin, H. S. Ghosh, K. L. Lewis, and V. Sisirak, "Plasmacytoid dendritic cells: recent progress and open questions," Annual Review of Immunology, vol. 29, pp. 163-183, 2011.

[52] V. Younesi, H. Nikzamir, M. Yousefi et al., "Epstein Barr virus inhibits the stimulatory effect of TLR7/8 and TLR9 agonists but not CD40 ligand in human B lymphocytes," Microbiology and Immunology, vol. 54, no. 9, pp. 534-541, 2010.

[53] I. Fathallah, P. Parroche, H. Gruffat et al., "EBV latent membrane protein 1 is a negative regulator of TLR9," The Journal of Immunology, vol. 185, no. 11, pp. 6439-6447, 2010.

[54] M. van Gent, B. D. Griffin, E. G. Berkhoff et al., "EBV lytic-phase protein BGLF5 contributes to TLR9 downregulation during productive infection," Journal of Immunology, vol. 186, no. 3, pp. 1694-1702, 2011.

[55] L. S. Young and A. B. Rickinson, "Epstein-Barr virus: 40 years on," Nature Reviews Cancer, vol. 4, no. 10, pp. 757-768, 2004.

[56] L. S. Young and P. G. Murray, "Epstein-Barr virus and oncogenesis: from latent genes to tumours," Oncogene, vol. 22, no. 33, pp. 5108-5121, 2003.

[57] S. Humme, G. Reisbach, R. Feederle et al., "The EBV nuclear antigen 1 (EBNA1) enhances B cell immortalization several thousandfold," Proceedings of the National Academy of Sciences of the United States of America, vol. 100, no. 19, pp. 10989-10994, 2003.

[58] S. P. Lee, J. M. Brooks, H. Al-Jarrah et al., "CD8 T cell recognition of endogenously expressed Epstein-Barr virus nuclear antigen 1," Journal of Experimental Medicine, vol. 199, no. 10, pp. 1409-1420, 2004.

[59] G. S. Taylor, H. M. Long, T. A. Haigh, M. Larsen, J. Brooks, and A. B. Rickinson, "A role for intercellular antigen transfer in the recognition of EBV-transformed B cell Lines by EBV nuclear antigen-specific $\mathrm{CD} 4^{+} \mathrm{T}$ cells," The Journal of Immunology, vol. 177, no. 6, pp. 3746-3756, 2006.

[60] D. Y. Lee and B. Sugden, "The latent membrane protein 1 oncogene modifies B-cell physiology by regulating autophagy," Oncogene, vol. 27, no. 20, pp. 2833-2842, 2008.
[61] Y. Chang, E. Cesarman, M. S. Pessin et al., "Identification of herpesvirus-like DNA sequences in AIDS-associated Kaposi's sarcoma," Science, vol. 266, no. 5192, pp. 1865-1869, 1994.

[62] R. Sun, S.-F. Lin, K. Staskus et al., "Kinetics of Kaposi’s sarcomaassociated herpesvirus gene expression," Journal of Virology, vol. 73, no. 3, pp. 2232-2242, 1999.

[63] F. X. Zhu, T. Cusano, and Y. Yuan, "Identification of the immediate-early transcripts of Kaposi's sarcoma-associated herpesvirus," Journal of Virology, vol. 73, no. 7, pp. 5556-5567, 1999.

[64] K. W. Wen and B. Damania, "Kaposi sarcoma-associated herpesvirus (KSHV): molecular biology and oncogenesis," Cancer Letters, vol. 289, no. 2, pp. 140-150, 2010.

[65] J. A. Ambroziak, D. J. Blackbourn, B. G. Herndier et al., "Herpes-like sequences in HIV-infected and uninfected Kaposi's sarcoma patients," Science, vol. 268, no. 5210, pp. 582-583, 1995.

[66] E. Cesarman, Y. Chang, P. S. Moore, J. W. Said, and D. M. Knowles, "Kaposi's sarcoma-associated herpesvirus-like DNA sequences in AIDS-related body-cavity-based lymphomas," The New England Journal of Medicine, vol. 332, no. 18, pp. 1186-1191, 1995.

[67] P. S. Moore and Y. Chang, "Kaposi's sarcoma-associated herpesvirus immunoevasion and tumorigenesis: two sides of the same coin?" Annual Review of Microbiology, vol. 57, pp. 609639, 2003.

[68] D. Ganem, "KSHV and the pathogenesis of Kaposi sarcoma: listening to human biology and medicine," The Journal of Clinical Investigation, vol. 120, no. 4, pp. 939-949, 2010.

[69] A. Carbone and A. Gloghini, "KSHV/HHV8-associated lymphomas," British Journal of Haematology, vol. 140, no. 1, pp. 1324, 2008.

[70] N. Dupin, C. Fisher, P. Kellam et al., "Distribution of human herpesvirus-8 latently infected cells in Kaposi's sarcoma, multicentric Castleman's disease, and primary effusion lymphoma," Proceedings of the National Academy of Sciences of the United States of America, vol. 96, no. 8, pp. 4546-4551, 1999.

[71] S. A. R. Rezaee, C. Cunningham, A. J. Davison, and D. J. Blackbourn, "Kaposi's sarcoma-associated herpesvirus immune modulation: an overview," Journal of General Virology, vol. 87, no. 7, pp. 1781-1804, 2006.

[72] D. Whitby, M. R. Howard, M. Tenant-Flowers et al., "Detection of Kaposi sarcoma associated herpesvirus in peripheral blood of HIV-infected individuals and progression to Kaposi's sarcoma," The Lancet, vol. 346, no. 8978, pp. 799-802, 1995.

[73] E. B. Quinlivan, C. Zhang, P. W. Stewart, C. Komoltri, M. G. Davis, and R. S. Wehbie, "Elevated virus loads of Kaposi's sarcoma-associated human herpesvirus 8 predict Kaposi's sarcoma disease progression, but elevated levels of human immunodeficiency virus type 1 do not," Journal of Infectious Diseases, vol. 185, no. 12, pp. 1736-1744, 2002.

[74] E. A. Engels, R. J. Biggar, V. A. Marshall et al., "Detection and quantification of Kaposi's sarcoma-associated herpesvirus to predict AIDS-associated Kaposi's sarcoma," AIDS, vol. 17, no. 12, pp. 1847-1851, 2003.

[75] D. M. Lukac and Y. Yuan, "Reactivation and lytic replication of KSHV," in Human Herpesviruses: Biology, Therapy, and Immunoprophylaxis, A. Arvin, G. Campadelli-Fiume, E. Mocarski et al., Eds., Cambridge University Press, Cambridge, UK, 2007. 
[76] C. Blasig, C. Zietz, B. Haar et al., "Monocytes in Kaposi's sarcoma lesions are productively infected by human herpesvirus 8," Journal of Virology, vol. 71, no. 10, pp. 7963-7968, 1997.

[77] W. J. Harrington Jr., O. Bagasra, C. E. Sosa et al., "Human herpesvirus type $8 \mathrm{DNA}$ sequences in cell-free plasma and mononuclear cells of Kaposi's sarcoma patients," Journal of Infectious Diseases, vol. 174, no. 5, pp. 1101-1105, 1996.

[78] M. C. Sirianni, L. Vincenzi, V. Fiorelli et al., "Gamma-interferon production in peripheral blood mononuclear cells and tumor infiltrating lymphocytes from Kaposi's sarcoma patients: correlation with the presence of human herpesvirus- 8 in peripheral blood mononuclear cells and lesional macrophages," Blood, vol. 91, no. 3, pp. 968-976, 1998.

[79] H. R. Lee, S. Lee, P. M. Chaudhary, P. Gill, and J. U. Jung, "Immune evasion by Kaposi's sarcoma-associated herpesvirus," Future Microbiology, vol. 5, no. 9, pp. 1349-1365, 2010.

[80] C. Boshoff and R. Weiss, "AIDS-related malignancies," Nature Reviews Cancer, vol. 2, no. 5, pp. 373-382, 2002.

[81] D. Ganem, "KSHV-induced oncogenesis," in Human Herpesviruses: Biology, Therapy, and Immunoprophylaxis, A. Arvin, G. Campadelli-Fiume, E. Mocarski et al., Eds., Cambridge University Press, Cambridge, UK, 2007.

[82] C. Liang, J.-S. Lee, and J. U. Jung, "Immune evasion in Kaposi's sarcoma-associated herpes virus associated oncogenesis," Seminars in Cancer Biology, vol. 18, no. 6, pp. 423-436, 2008.

[83] K. Brulois and J. U. Jung, "Interplay between Kaposi's sarcoma-associated herpesvirus and the innate immune system," Cytokine \& Growth Factor Reviews, vol. 25, no. 5, pp. 597609, 2014.

[84] M. S. Lee, T. Jones, D. Y. Song et al., "Exploitation of the complement system by oncogenic Kaposi's sarcoma-associated herpesvirus for cell survival and persistent infection," PLoS Pathogens, vol. 10, no. 9, Article ID e1004412, 2014.

[85] Z. Hu and E. J. Usherwood, "Immune escape of $\gamma$-herpesviruses from adaptive immunity," Reviews in Medical Virology, vol. 24, no. 6, pp. 365-378, 2014.

[86] J. A. West, S. M. Gregory, V. Sivaraman, L. Su, and B. Damania, "Activation of Plasmacytoid dendritic cells by Kaposi's sarcomaassociated herpesvirus," Journal of Virology, vol. 85, no. 2, pp. 895-904, 2011.

[87] G. Rappocciolo, F. J. Jenkins, H. R. Hensler et al., "DC-SIGN is a receptor for human herpesvirus 8 on dendritic cells and macrophages," Journal of Immunology, vol. 176, no. 3, pp. 17411749, 2006.

[88] H. R. Hensler, G. Rappocciolo, C. R. Rinaldo, and F. J. Jenkins, "Cytokine production by human herpesvirus 8-infected dendritic cells," Journal of General Virology, vol. 90, no. 1, pp. 79-83, 2009.

[89] M. Cirone, V. Conte, A. Farina et al., "HHV-8 reduces dendritic cell migration through down-regulation of cell-surface CCR6 and CCR7 and cytoskeleton reorganization," Virology Journal, vol. 9, article 92, 2012.

[90] D. M. Campbell, G. Rappocciolo, F. J. Jenkins, and C. R. Rinaldo, "Dendritic cells: key players in human herpesvirus 8 infection and pathogenesis," Frontiers in Microbiology, vol. 5, article 452, 2014.

[91] S. Della Bella, S. Nicola, L. Brambilla et al., "Quantitative and functional defects of dendritic cells in classic Kaposi's sarcoma," Clinical Immunology, vol. 119, no. 3, pp. 317-329, 2006.

[92] J. Stebbing, B. Gazzard, S. Portsmouth et al., "Disease-associated dendritic cells respond to disease-specific antigens through the common heat shock protein receptor," Blood, vol. 102, no. 5, pp. 1806-1814, 2003.

[93] F. Valcuende-Cavero, M. I. Febrer-Bosch, and A. CastellsRodellas, "Langerhans' cells and lymphocytic infiltrate in AIDSassociated Kaposi's sarcoma. An immunohistochemical study," Acta Dermato-Venereologica, vol. 74, no. 3, pp. 183-187, 1994.

[94] J. van Grol, C. Subauste, R. M. Andrade, K. Fujinaga, J. Nelson, and C. S. Subauste, "HIV-1 inhibits autophagy in bystander macrophage/monocytic cells through Src-Akt and STAT3," PLoS ONE, vol. 5, no. 7, Article ID el1733, 2010.

[95] S. Shen, M. Niso-Santano, S. Adjemian et al., "Cytoplasmic STAT3 represses autophagy by inhibiting PKR activity," Molecular Cell, vol. 48, no. 5, pp. 667-680, 2012.

[96] R. Santarelli, R. Gonnella, G. di Giovenale et al., "STAT3 activation by KSHV correlates with IL-10, IL-6 and IL-23 release and an autophagic block in dendritic cells," Scientific Reports, vol. 4, article 4241, 2014.

[97] L. W. Thomas, C. Lam, and S. W. Edwards, "Mcl-1; the molecular regulation of protein function," FEBS Letters, vol. 584, no. 14, pp. 2981-2989, 2010.

[98] W. T. Tai, C. W. Shiau, H. L. Chen et al., "Mcl-1-dependent activation of Beclin 1 mediates autophagic cell death induced by sorafenib and SC-59 in hepatocellular carcinoma cells," Cell Death and Disease, vol. 4, no. 2, article e485, 2013.

[99] E. H.-Y. Cheng, J. Nicholas, D. S. Bellows et al., "A Bcl-2 homolog encoded by Kaposi sarcoma-associated virus, human herpesvirus 8, inhibits apoptosis but does not heterodimerize with Bax or Bak," Proceedings of the National Academy of Sciences of the United States of America, vol. 94, no. 2, pp. 690694, 1997.

[100] J.-S. Lee, Q. Li, J.-Y. Lee et al., "FLIP-mediated autophagy regulation in cell death control," Nature Cell Biology, vol. 11, no. 11, pp. 1355-1362, 2009.

[101] S. Q. Crome, A. Y. Wang, and M. K. Levings, “Translational mini-review series on Th17 cells: function and regulation of human $\mathrm{T}$ helper 17 cells in health and disease," Clinical \& Experimental Immunology, vol. 159, no. 2, pp. 109-119, 2010.

[102] Y. Nefedova, M. Huang, S. Kusmartsev et al., "Hyperactivation of STAT3 is involved in abnormal differentiation of dendritic cells in cancer," Journal of Immunology, vol. 172, no. 1, pp. 464474, 2004.

[103] U. Bharadwaj, M. Li, R. Zhang, C. Chen, and Q. Yao, "Elevated interleukin-6 and G-CSF in human pancreatic cancer cell conditioned medium suppress dendritic cell differentiation and activation," Cancer Research, vol. 67, no. 11, pp. 5479-5488, 2007.

[104] M. Kortylewski, H. Xin, M. Kujawski et al., "Regulation of the IL-23 and IL-12 balance by Stat3 signaling in the tumor microenvironment," Cancer Cell, vol. 15, no. 2, pp. 114-123, 2009.

[105] M. Djerbi, V. Screpanti, A. I. Catrina, B. Bogen, P. Biberfeld, and A. Grandien, "The inhibitor of death receptor signaling, FLICEinhibitory protein defines a new class of tumor progression factors," Journal of Experimental Medicine, vol. 190, no. 7, pp. 1025-1031, 1999.

[106] I. Guasparri, S. A. Keller, and E. Cesarman, "KSHV vFLIP is essential for the survival of infected lymphoma cells," The Journal of Experimental Medicine, vol. 199, no. 7, pp. 993-1003, 2004.

[107] A. M. Leidal, D. P. Cyr, R. J. Hill, P. W. K. Lee, and C. McCormick, "Subversion of autophagy by Kaposi's sarcomaassociated herpesvirus impairs oncogene-induced senescence," Cell Host and Microbe, vol. 11, no. 2, pp. 167-180, 2012. 
[108] C. Liang, "Viral FLIPping autophagy for longevity," Cell Host and Microbe, vol. 11, no. 2, pp. 101-103, 2012.

[109] S. Koopal, J. H. Furuhjelm, A. Järviluoma et al., "Viral oncogene-induced DNA damage response is activated in Kaposi sarcoma tumorigenesis," PLoS Pathogens, vol. 3, no. 9, pp. 13481360, 2007.

[110] R. Sarid, T. Sato, R. A. Bohenzky, J. J. Russo, and Y. Chang, "Kaposi's sarcoma-associated herpesvirus encodes a functional Bcl-2 homologue," Nature Medicine, vol. 3, no. 3, pp. 293-298, 1997.

[111] Q. Liang, B. Chang, K. F. Brulois et al., "Kaposi's sarcomaassociated herpesvirus $\mathrm{K} 7$ modulates rubicon-mediated inhibition of autophagosome maturation," Journal of Virology, vol. 87, no. 22, pp. 12499-12503, 2013.

[112] H.-W. Wang, T. V. Sharp, A. Koumi, G. Koentges, and C. Boshoff, "Characterization of an anti-apoptotic glycoprotein encoded by Kaposi's sarcoma-associated herpesvirus which resembles a spliced variant of human survivin," The EMBO Journal, vol. 21, no. 11, pp. 2602-2615, 2002.

[113] P. Feng, J. Park, B.-S. Lee, S.-H. Lee, R. J. Bram, and J. U. Jung, "Kaposi's sarcoma-associated herpesvirus mitochondrial K7 protein targets a cellular calcium-modulating cyclophilin ligand to modulate intracellular calcium concentration and inhibit apoptosis," Journal of Virology, vol. 76, no. 22, pp. 1149111504, 2002.

[114] M. Mehrpour, A. Esclatine, I. Beau, and P. Codogno, "Overview of macroautophagy regulation in mammalian cells," Cell Research, vol. 20, no. 7, pp. 748-762, 2010.

[115] K. Matsunaga, T. Saitoh, K. Tabata et al., "Two Beclin 1-binding proteins, Atg14L and Rubicon, reciprocally regulate autophagy at different stages," Nature Cell Biology, vol. 11, no. 4, pp. 385396, 2009.

[116] Y. Zhong, Q. J. Wang, X. Li et al., "Distinct regulation of autophagic activity by Atg14L and Rubicon associated with Beclin 1-phosphatidylinositol-3-kinase complex," Nature Cell Biology, vol. 11, no. 4, pp. 468-476, 2009. 


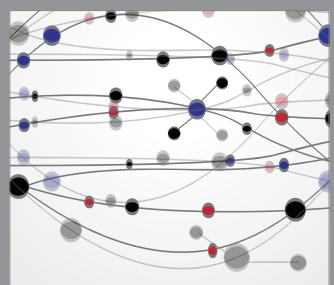

The Scientific World Journal
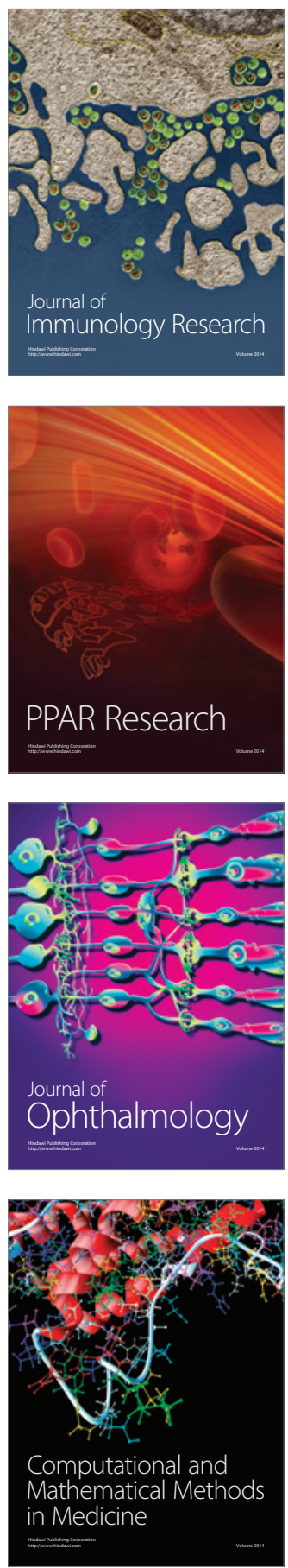

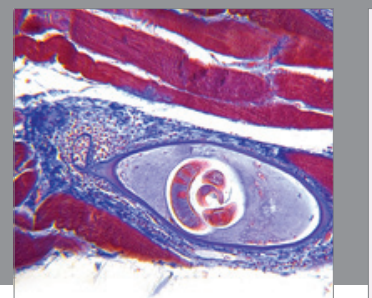

Gastroenterology

Research and Practice
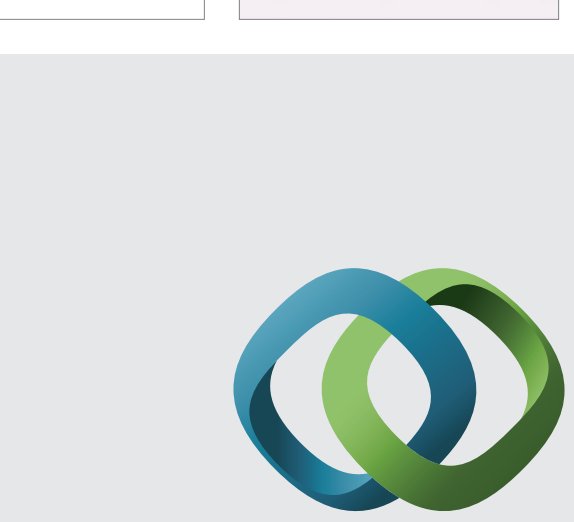

\section{Hindawi}

Submit your manuscripts at

http://www.hindawi.com
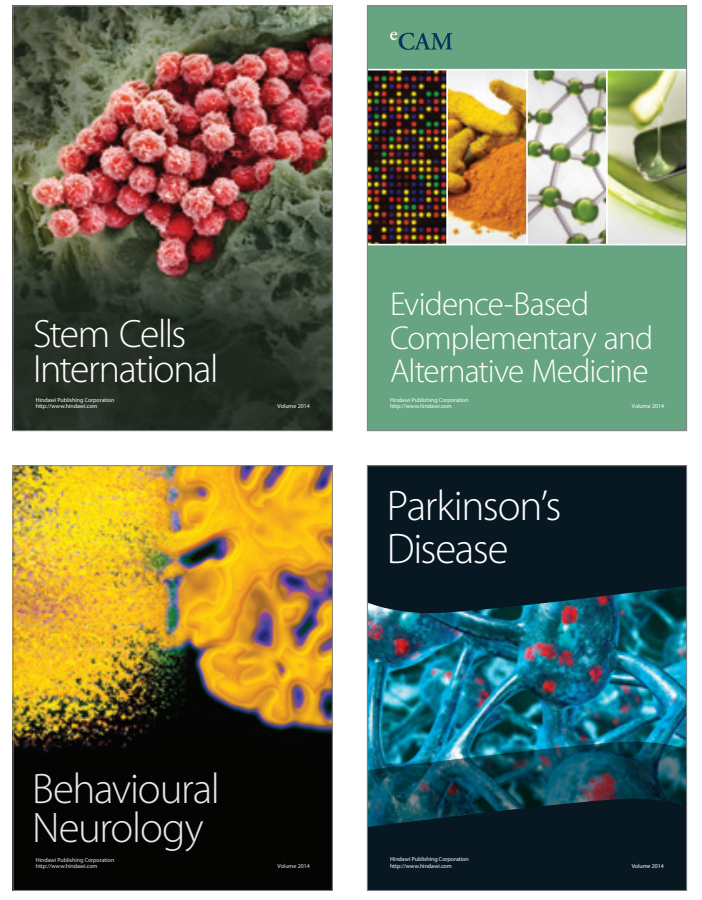
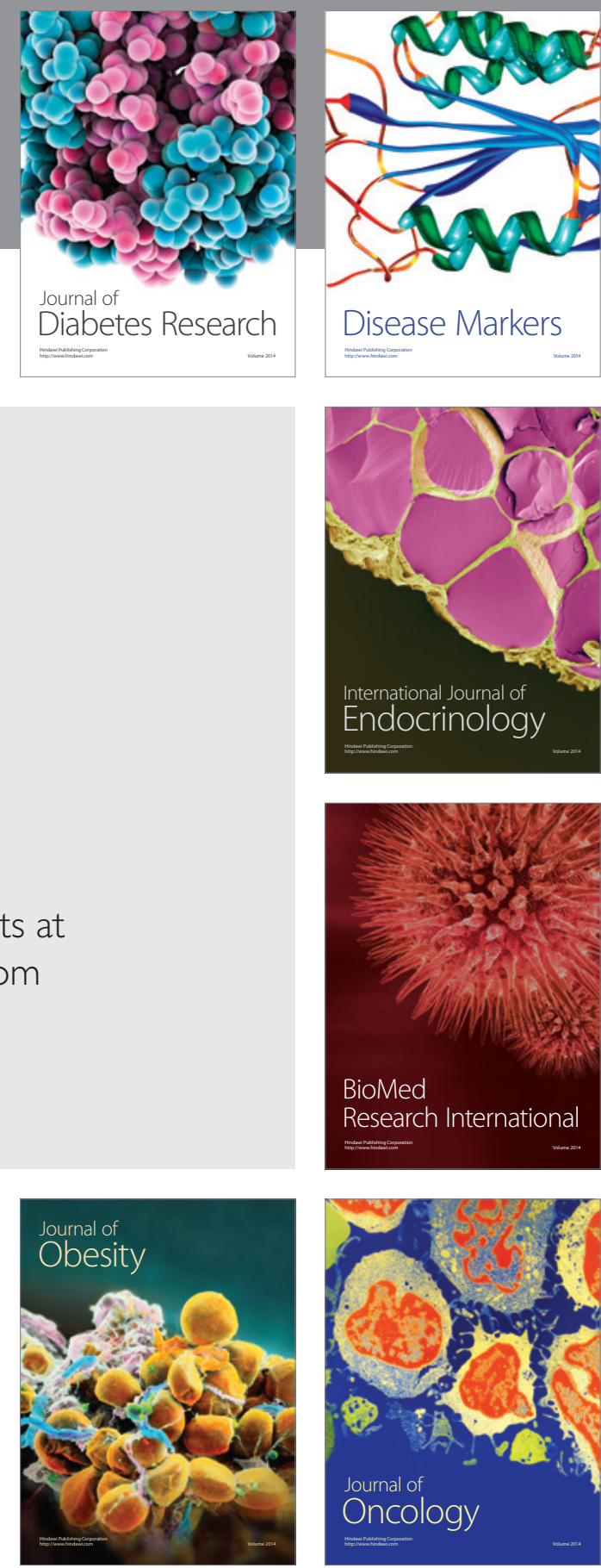

Disease Markers
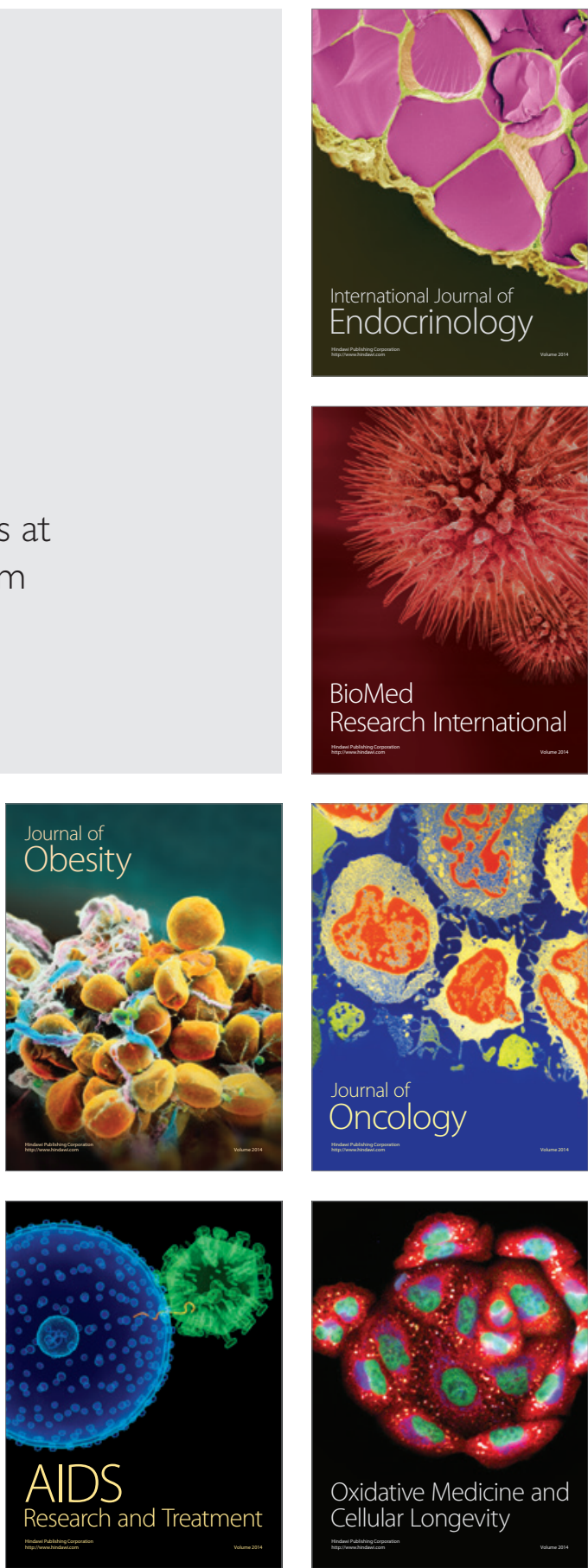